\title{
Zn-catalyzed tert-butyl nicotinate-directed amide cleavage for applications in peptide synthesis and peptidomimetic design
}

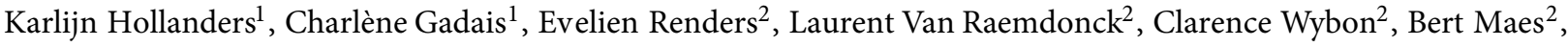 \\ Steven Ballet ${ }^{1}$ \\ ${ }^{1}$ Research Group of Organic Chemistry, Departments of Chemistry and Bioengineering Sciences, Vrije Universiteit Brussel, Belgium \\ ${ }^{2}$ Organic Synthesis, Department of Chemistry, University of Antwerp, Belgium
}

https://doi.org/10.17952/35EPS.2018.072

\section{Introduction}

The amide bond is the key functional group that makes up peptides and proteins. Consequently, the selective formation of this bond has been extensively studied by peptide scientists with the development of a wellestablished catalog of so-called coupling reagents as a result, and the general method remains the direct acylation of an amine by means of an in situ activated carboxylic acid.[1] To date, amides have rarely been considered as a carboxylic acid surrogate because of its poor electrophilicity and intrinsic stability. Recently, we presented the transformation of primary amides into esters through use of a tert-butyl nicotinate directing group.[2] Logically, the amide to ester conversion represented the ideal precedent to attempt transamidation reactions in a next step.

The activation mechanism of the amide can be considered as a 'biomimetic' protocol: the C3-ester substituent of the pyridine in the directing group populates a trans amide conformer suitable for $\mathrm{Zn}$-chelation, $\mathrm{C}=\mathrm{O}_{\text {amide }}{ }^{-}$

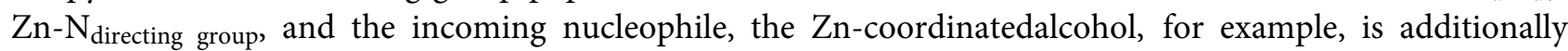
activated as a nucleophile by hydrogen bonding with the acetate ligand of the catalyst. Additionally, the acetate ligand assists in an intramolecular $\mathrm{O}-$ to-N proton transfer.

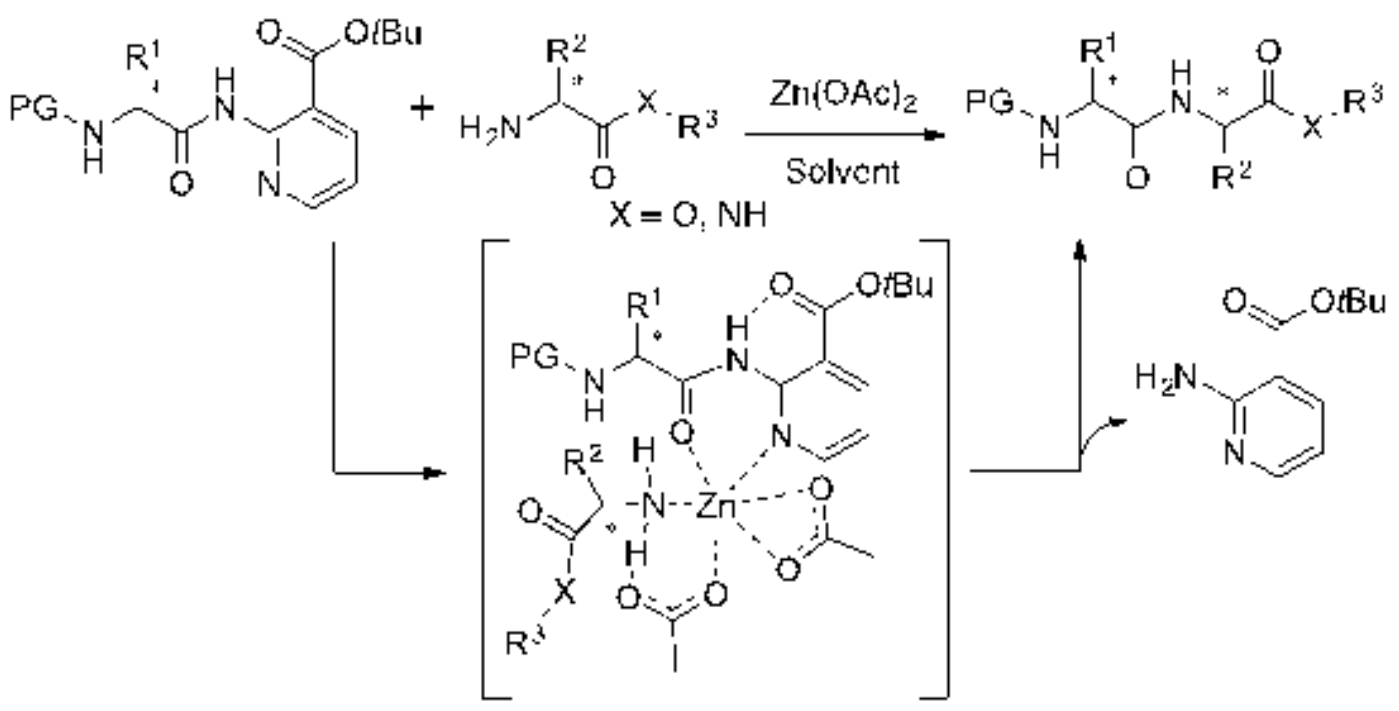

Figure 1: Peptide Coupling by Transamidation

We developed reaction conditions which allow the use of the directing group activated amino acid amides in transamidation reactions involving the $\alpha$-amines of amino acid esters/amides as a method for diverse applications in peptide research. Next to amino acid - amino acid couplings, we exemplify the methodology's utility on more complex systems by segment condensations and macrocyclisations.[3]

\section{Results and Discussion}

The directing group is introduced via a previously developed Pd-catalyzed amidation protocol onto the N-Bocprotected amino acid amides. Here, tert-butyl 2-chloronicotinate 2 is coupled to the primary amides in the presence of 3rd generation palladacycle with a dcpf-ligand 3 and a carbonate base (Table 1). 


\begin{tabular}{|c|c|c|}
\hline (1.1 equiv) & $\begin{array}{c}\text { Pd.G3-dcpt } 3 \text { (5 mol\%) }) \\
\mathrm{Cs}_{2} \mathrm{CO}_{3}(1.1 \text { equiv) } \\
2-\mathrm{MeTHF}^{2}(0.16 \mathrm{M}) \\
40^{\circ} \mathrm{C}, 24 \mathrm{~h}\end{array}$ & Pd-G3-dcpt \\
\hline Entry & Boc-AA-NH-tBu nic 4 & Yield [\%] [a] \\
\hline 1 & Boc-L-Phe-NH-tBu nic L-4a & $93^{[b]}$ \\
\hline 2 & Boc-L-Ala-NH-tBu nic L-4b & $71^{[b]}$ \\
\hline 3 & Boc-L-Tyr(tBu)-NH-tBu nic L-4c & $94^{[b]}$ \\
\hline 4 & Boc-L-Pro-NH-tBu nic L-4d & $80^{[c]}$ \\
\hline 5 & Boc-L-Met-NH-tBu nic L-4e & $85^{[b, d]}$ \\
\hline 6 & Boc-BAla-NH-tBu nic L-4f & 99 \\
\hline 7 & Boc-L-Val-NH-tBu nic L-4h & $98^{[\mathrm{e}]}$ \\
\hline 8 & Boc-L-lle-NH-tBu nic L-4i & 99[e] \\
\hline 9 & Boc-L-Ser(tBu)-NH-tBu nic $\mathbf{L}-4 \mathbf{j}$ & $72^{[b, f]}$ \\
\hline
\end{tabular}

[a] Isolated yield. [b] K2CO3 (7.5 equiv), Pd-G3-dcpf (10 mol\%) in 1,4-dioxane, 50 ${ }^{\circ} \mathrm{C}, 30 \mathrm{~h}$. [c] Cs2 $\mathrm{CO} 3(1.2$ equiv). [d] $60^{\circ} \mathrm{C}$. [e] $\mathrm{Cs} 2 \mathrm{CO} 3$ (2 equiv). [f] $\mathrm{K} 2 \mathrm{CO} 3$ (6 equiv).

For transamidations yielding 'peptide bonds', the $t \mathrm{Bu}$ nic amino acid amides 4 were used in the presence of 20 $\mathrm{mol} \% \mathrm{Zn}(\mathrm{OAc})_{2}$ at $70^{\circ} \mathrm{C}$ for $24 \mathrm{~h}$ in THF. Since $\alpha$-amino esters of type 5 , used herein as a nucleophile in slight excess (1.1 equivalents), are often sold as $\mathrm{HCl}$ salt, $\mathrm{NaOAc}$ was added in a 1:1 ratio to liberate the free amine in situ. Under these conditions, the synthesis of dipeptides was realized (Table 2). The transamidation tolerated variable sterical hindrance in the lateral chains, as shown for both the $\mathrm{tBu}$ nic amino acid amides and the amino acid-based nucleophiles. Regarding the $\alpha$-amino ester functionality, methyl, allyl, tert-butyl and benzyl esters remain unaffected. For amino acid amides as the nucleophile, featuring a primary amide, 3 equivalents were required to obtain full conversion. A similar observation was made for $\mathrm{H}-\mathrm{L}-\mathrm{Pro}-\mathrm{OMe}$.HCl. In the latter case, however, an increase in temperature to $100^{\circ} \mathrm{C}$ was additionally required to obtain full conversion within $24 \mathrm{~h}$.

Table 2: Scope of the transamidation reaction for the synthesis of various dipeptides

\begin{tabular}{|c|c|c|c|c|}
\hline & $\mathrm{HCl}_{2}^{\mathrm{H}_{2} \mathrm{~N}}$ & $\begin{array}{c}\mathrm{Zn}(\mathrm{OAc})_{2}(20 \mathrm{~mol} \%) \\
\mathrm{NaOAc}(1.1 \text { equiv) } \\
\mathrm{THF}(C 0.5 \mathrm{M}) \\
70^{\circ} \mathrm{C}, 24 \mathrm{~h}\end{array}$ & 1 & 이, \\
\hline Entry & Boc-AA-NH-tBu nic 4 & Amine $\mathbf{5 . H C l}$ & Product & Yield [\%] ${ }^{[\mathrm{a}]}$ \\
\hline 1 & Boc-L-Phe-NH-tBu nic L-4a & H-L-Phe-OMe.HCl L-5a.HCl & $L, L-6 a$ & 95 \\
\hline 2 & Boc-L-Ala-NH- $t$ Bu nic $\mathrm{L}-\mathbf{4 b}$ & H-L-Leu-OAll.HCl L-5b.HCl & $L, L-6 b$ & 99 \\
\hline 3 & Boc-L-Tyr(tBu)-NH-tBu nic L-4c & $\mathrm{H}-\mathrm{L}-\mathrm{Val}-\mathrm{O} t \mathrm{Bu} . \mathrm{HCl} \mathrm{L}-5 \mathrm{c} . \mathrm{HCl}$ & $L, L-6 c$ & 83 \\
\hline 4 & Boc-L-Pro-NH-tBu nic L-4d & H-L-Leu-OMe. HCl l-5d. $\mathbf{H C l}$ & $L, L-6 d$ & 95 \\
\hline 5 & Boc-L-Met-NH-tBu nic L-4e & H-L-Trp-OBn.HCl L-5e.HCl & $L, L-6 e$ & 80 \\
\hline 7 & Boc- $\beta$ Ala-NH-tBu nic L-4f & $\mathrm{H}-\mathrm{L}-\mathrm{Phe}-\mathrm{NH}_{2} \cdot \mathrm{HCl} \mathrm{L}-5 \mathrm{f} . \mathrm{HCl}$ & $L, L-6 f$ & $74^{[\mathrm{b}]}$ \\
\hline 8 & Boc-L-Met-NH-tBu nic $\mathbf{L}-\mathbf{4 e}$ & H-L-Phe-NH $2 . \mathrm{HCl}$ L-5f.HCl & $L, L-6 g$ & $89^{[\mathrm{b}]}$ \\
\hline 9 & Boc-L-Ala-NH-tBu nic L-4b & $\mathrm{H}-\mathrm{L}-$ Pro-OMe. $\mathrm{HCl}$ L-5g. $\mathrm{HCl}$ & $L, L-6 h$ & $68^{[b, c]}$ \\
\hline
\end{tabular}

[a] Isolated yield; [b] 3 equiv of the amine; $[\mathrm{c}] \mathrm{T}=100^{\circ} \mathrm{C}$

Upon use of the reported transamidation in segment condensations and cyclizations, the N-Boc tBu nic amino acid amides were used as building blocks in peptide synthesis (e.g., synthesis of L,L-7 in Scheme 1). The Boc deprotection was performed using $\mathrm{HCl}$ in dioxane, followed by a coupling in solution with EDC$\mathrm{HOBt}$, and resulting in the dipeptide in excellent yield without loss of our directing group. 

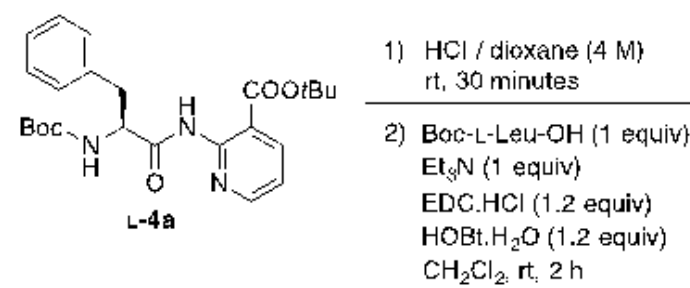<smiles>CCOC(=O)c1cccnc1NC(=O)CNC(=O)C(CC(C)C)NC(=O)OCc1ccccc1</smiles>

$96 \%$

Scheme 1: Use of N-Boc tert-butyl nicotinate amino acid amide in peptide synthesis

Following the dipeptide synthesis, the transamidations were evaluated for the assembly of small segments. The $t \mathrm{Bu}$ nic dipeptides 1,17 and 1,1-8 were engaged in a transamidation with, respectively, HlLeu-l-ValOtBu.HCl (1,1-9.HCl) and H-l-Ala-l-Phe-OtBu.HCl (1,1-10.HCl) under standard conditions (Scheme 2a). Both tetramers L,L-11 and L,L-12 could be isolated in a very good yield.

a. Segment condensation
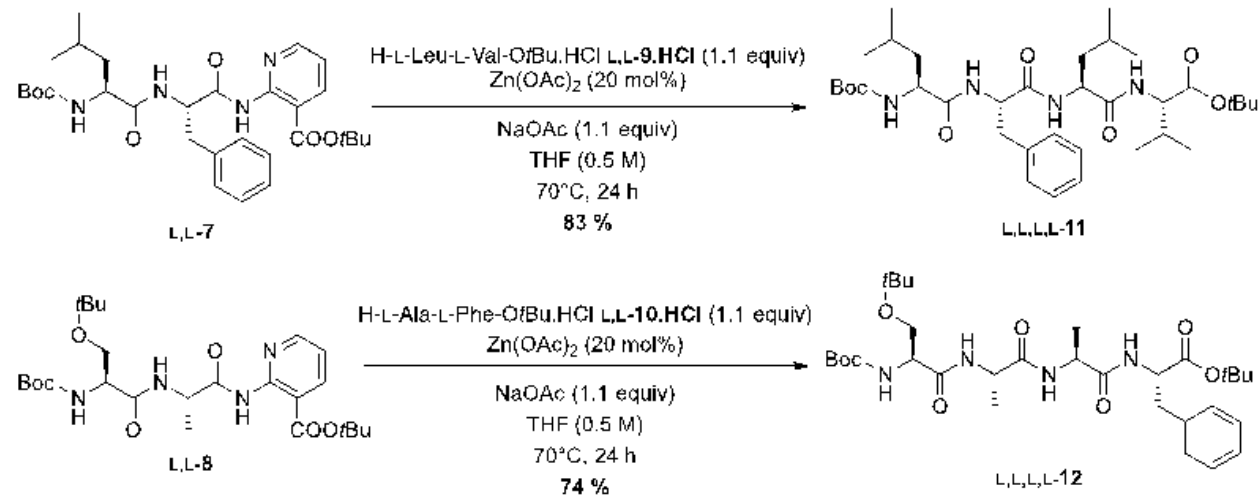

b. Cyclization
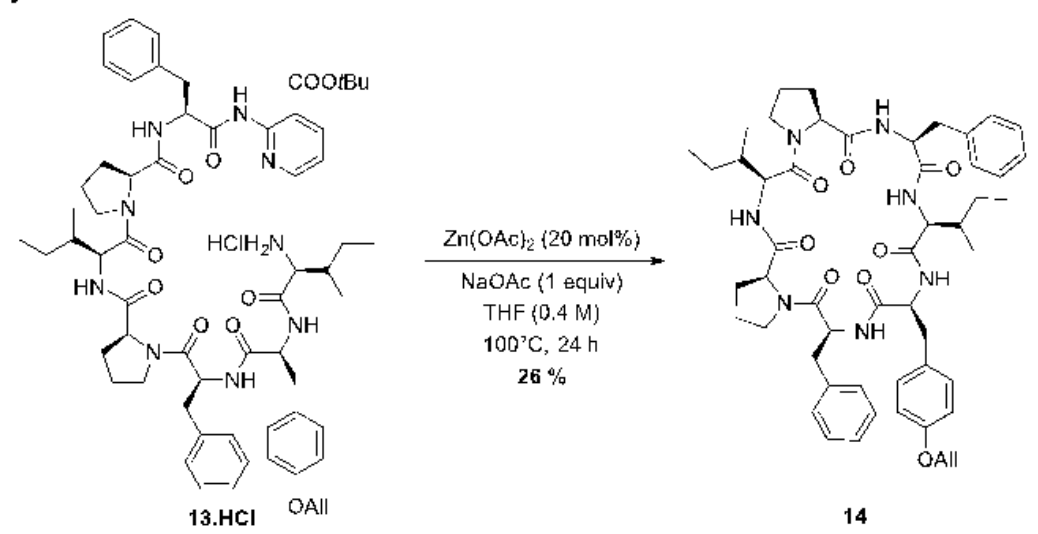

14

Scheme 2: Application of the methodology on a) dipeptide segment condensations and b) the macrocyclization of $13 . \mathrm{HCl}$

Macrocyclization was demonstrated by the synthesis of an allylated analog of Stylissatin A. Similar to the segment syntheses, the linear heptamer $13 . \mathrm{HCl}$ was prepared starting from $\mathrm{N}$-Boc protected $\mathrm{tBu}$ nic phenylalanine in solution under classical coupling/deprotection conditions. The cyclization was achieved smoothly within 24 hours at $100^{\circ} \mathrm{C}$, allowing the isolation of the cyclic heptamer 14 by means of preparative HPLC purification (Scheme 2b).

\section{Conclusion}

To conclude, we have developed a transamidation protocol, proceeding under mild conditions, that is applicable on amino acids and small peptides. As the $\mathrm{N}$-Boc protected $t \mathrm{Bu}$ nic amino acids amides are compatible with standard deprotection/coupling conditions, this methodology has added to the chemical toolbox of peptide 
chemists. Importantly, the mild condensation and cyclization conditions are orthogonal to standard peptide synthesis conditions

\section{Acknowledgements}

This work was financially supportedby the Research FoundationFlanders (FWO; Research Project and Scientific Research Network (WOG)), the Hercules Foundationand the University of Antwerp (BOF).

\section{References}

[1] a) A. El-Faham, F. Albericio, Chem. Rev. 2011, 111, 6557-6602; DOI: 10.1021/cr100048w b) F. Albericio, A. El-Faham, Org. Process Res. Dev. 2018, 22, 760-772; DOI: 10.1021/acs.oprd.8b00159.

[2] C. C. D. Wybon, C. Mensch, K. Hollanders, C. Gadais, W. A. Herrebout, S. Ballet, B. U. W. Maes, ACS Catal. 2018, 8, 203-218; DOI: 10.1021/acscatal.7b02599

[3] K. Hollanders, C. Gadais, E. Renders, L. Van Raemdonck, C.C. D. Wybon, D. Masullo, B. U. W. Maes, S. Ballet, Sumitted. 